\title{
Various therapeutic effects of immune checkpoint inhibitors
}

\author{
Noriomi Matsumura ${ }^{1}$
}

Published online: 11 August 2020

(c) The Japan Society of Clinical Oncology 2020

In this issue, 3 case reports suggest how to determine which patients to select for and how to use immune checkpoint inhibitors.

Masago et al. reported a case of 68-year old man who had Xp11.2 translocation renal cell carcinoma (RCC) with TFE3 gene fusion [1]. This tumor is extremely rare in the elderly. The patient was treated with nivolumab and ipilimumab and had poor response and then was treated with axitinib, but eventually died 6 months after surgery. In general, immune checkpoint inhibitors are effective for RCC, but they may not be effective for this type of tumor.

Doi et al. reported a case in which nivolumab was administered for chemotherapy-resistant gastric cancer and showed a marked effect [2]. Interestingly, the patient continued to have a long-term antitumor effect after discontinuing nivolumab due to an immune-related adverse event (irAE). This case suggests that there is no need for repeated use of immune checkpoint inhibitors, because once patients respond to immune checkpoint inhibitors, their tumor immunity is memorized. Future clinical trials to determine when immune checkpoint inhibitor therapy ends will be necessary to prevent irAEs and establish economically acceptable treatment strategies, but it is important to accumulate such case reports first.

Kuhara et al. treated unresectable gastric cancer with chemotherapy followed by surgery and radiotherapy while nivolumab was administered [3]. Tumors in non-irradiated areas also shrank. This may be the so-called abscopal effect of irradiation to activate tumor immunity, which may have strengthened the effect of nivolumab. However, it is difficult to say whether the irradiation truly activated tumor immunity or whether nivolumab did so without irradiation. However, there is no doubt that nivolumab did prolong survival. Immune checkpoint inhibitors may be more effective in combination with existing cancer therapies than as single agents, and in view of this, a number of clinical trials are underway, which will significantly change the future of cancer treatment.

\section{References}

1. Masago T, Kobayakawa S, Ohtani Y et al (2020) Xp11.2 translocation renal cell carcinoma with TFE3 gene fusion in the elderly: case report and literature review. Int Canc Conf J. https://doi. org/10.1007/s13691-020-00430-6

2. Doi H, Ninomiya M, Toyota K et al (2020) A case of multiple metastatic gastric cancer with primary lesion vanished after administrating nivolumab, and the effect remains even after discontinuance of therapy. Int Canc Conf J. https://doi.org/10.1007/ s13691-020-00432-4

3. Kuhara Y, Ninomiya M, Hirahara S et al (2020) A long-term survival case of unresectable gastric cancer with multidisciplinary therapy including immunotherapy and abscopal effect. Int Canc Conf J. https://doi.org/10.1007/s13691-020-00433-3

Publisher's Note Springer Nature remains neutral with regard to jurisdictional claims in published maps and institutional affiliations.
Noriomi Matsumura

noriomi@med.kindai.ac.jp

1 Faculty of Medicine, Kindai University, Osakasayama, Japan 